\title{
Del síntoma a la enfermedad: dolor abdominal recurrente
}

\author{
M. Enrubia Iglesias \\ Pediatra. CAP Sants. Institut Català de la Salut. Barcelona. España.
}

Rev Pediatr Aten Primaria. 2009; I I (Supl I 7):s437-s44 I

Manel Enrubia Iglesias, menrubia.bcn.ics@gencat.cat

Resumen

El dolor abdominal recurrente es un motivo de consulta frecuente en el ámbito de la Pediatría de Atención Primaria. Se presenta un caso clínico de dolor abdominal recidivante, cuyo diagnóstico final fue de pancreatitis aguda. Se expone la anamnesis, exploración física, diagnóstico diferencial, exploraciones complementarias, así como un resumen de la pancreatitis aguda en la edad pediátrica.

Palabras clave: Dolor abdominal, Pancreatitis aguda, Colelitisiasis, Adolescente.

Abstract

Recurrent abdominal pain is a motive of frequent consultation in paediatrics Primary Care.

A clinical case of recurrent abdominal pain is presented in which the final diagnosis was acute pancreatitis. The anamnesis, physical examination, complementary test, differential diagnosis, as well as a summary of acute pancreatitis in childhood is also discussed.

Key words: Abdominal pain, Acute pancreatitis, Cholelithiasis, Teenager.

\section{Introducción}

El dolor abdominal recurrente es un motivo de consulta frecuente en el ámbito de la Pediatría de Atención Primaria, que puede ser debido a numerosas causas, tanto orgánicas como funcionales.

Una cuidadosa anamnesis y exploración física nos ayudarán a orientar el po- sible origen orgánico o, por eliminación, funcional.

El caso clínico corresponde a una adolescente que consulta por dolor abdominal agudo. La anamnesis nos permite orientarlo como dolor abdominal recidivante y llegar al diagnóstico etiológico de una entidad potencialmente grave de un modo más

El autor declara no presentar posibles conflictos de intereses en relación con la preparación y publicación de este artículo. 
rápido, resultando un beneficio para la paciente.

El caso clínico nos permite revisar los aspectos más relevantes del diagnóstico diferencial y el uso de las exploraciones complementarias, así como revisar la pancreatitis aguda en la edad pediátrica.

\section{Caso clínico}

Paciente de sexo femenino de 13 años y 7 meses de edad que consulta por dolor abdominal y vómitos de 2 días de evolución.

Antecedentes familiares: sin interés. Antecedentes personales: origen boliviano. Hace 3 semanas que ha llegado a Barcelona por reagrupación familiar (su madre lleva en Barcelona 5 años). Menarquia hace 4 meses. No patologías previas de relevancia.

Enfermedad actual: dolor abdominal de 2-3 días de evolución, difuso, irradiado a epigastrio, que en ocasiones se acompaña de vómitos. No alteración del ritmo deposicional. Acudió a un servicio de urgencias donde se le pautó ranitidina y omeprazol. Acude por persistencia del cuadro. La paciente refiere que, estando en su país, ha presentado 2 cuadros de dolor abdominal aislado en los últimos 2 meses, que ha relacionado con la menstruación. Espera la menstruación en estos días. Se trata pues de un dolor abdominal recurrente.

Exploración física: afebril, buen estado general, buena hidratación de piel y mucosas, buena coloración. Obesidad troncular. Abdomen blando y depresible. Dolor abdominal difuso a la palpación en epigastrio y flancos. No se palpan masas abdominales. Peristaltismo normal. Puño percusión lumbar: negativa. Auscultación cardiorrespiratoria normal. ORL: normal. Neurológico normal.

Exploraciones complementarias: una vez orientado el caso con los datos de la historia clínica, las pruebas nos ayudarán a eliminar o confirmar la causa orgánica. En nuestro caso se inició el estudio con una analítica sanguínea y ecografía abdominal que mostraron: Hto $37,8 \% \mathrm{Hb} 13,4 \mathrm{~g} / \mathrm{dl}$, plaquetas $338.000 / \mathrm{ml}$, leucocitos $7.700 / \mathrm{ml}$ (N $46 \%)$; glucosa $79 \mathrm{mg} / \mathrm{dl}$, creatinina 0,55 mg/dl, proteínas $77 \mathrm{~g} / \mathrm{l}$, albúmina $41 \mathrm{gl} / \mathrm{l}$, bilirrubina total $2,1 \mathrm{mg} / \mathrm{dl}$, colesterol $206 \mathrm{mg} / \mathrm{dl}$, ALT $475 \mathrm{UI} / \mathrm{L}$, AST $215 \mathrm{UI} / \mathrm{L}$, GGT $243 \mathrm{UI} / \mathrm{L}$, amilasas 427,8 UI/L, lipasa 1298UI/L. Ecografía abdominal: vía biliar poco distendida con imagen de litiasis de 15 mm. Páncreas no valorable por interposición de gas intestinal.

Diagnóstico final y evolución: se trata pues, de un caso de pancreatitis aguda 
secundaria a litiasis biliar. Se procedió a ingreso hospitalario, dándose de alta al cabo de una semana. A los 5 días del alta presentó un nuevo episodio del mismo cuadro, por lo que volvió a ingresar y se aceleró la programación de una colecistectomía, tras la cual la paciente no ha vuelto a presentar episodios de dolor abdominal.

\section{Discusión}

El dolor abdominal es un motivo de consulta frecuente en el ámbito de la Pediatría de Atención Primaria. Aproximadamente, dos terceras partes de los casos son de origen médico, el resto tiene un origen quirúrgico. Un tercio de los dolores abdominales de origen médico quedan sin diagnóstico específico ${ }^{1}$. Afectan predominantemente a niñas entre 4 y 6 años y en los primeros años de la adolescencia ${ }^{2}$.

Para una correcta orientación es imprescindible una cuidadosa anamnesis (características de dolor, tiempo de evolución, número de episodios similares) y una detallada exploración física (desde la existencia de signos que orienten a un cuadro quirúrgico, a la normalidad en la exploración que nos oriente a un dolor abdominal funcional) ${ }^{1,3,4}$. En nuestro caso se trata de un dolor abdominal recurrente sin signos sugestivos de abdomen agudo.
En el diagnóstico diferencial del dolor abdominal recurrente consideraremos como causas más frecuentes ${ }^{1,3}$ :

- Causas orgánicas: estreñimiento; intolerancia a la lactosa; parasitosis intestinales (especialmente por Giardia lamblia); gastritis por Helicobacter pylori; úlcera gastroduodenal; tumoraciones abdominales; enfermedad de Crohn; causas ginecológicas: dolores de ovulación, hematocolpos; litiasis urinaria; litiasis biliar; pancreatitis.

- Causas funcionales: dispepsia funcional, síndrome del intestino irritable, dolor abdominal funcional.

Las exploraciones complementarias nos ayudarán en el diagnóstico, una vez hayamos orientado el dolor abdominal mediante la anamnesis y la exploración física. Las exploraciones iniciales a considerar comprenden: analítica sanguínea (hemograma con VSG, glucemia, función hepática y renal, amilasa, lipasa), ecografía abdominal, analítica de orina, investigación parásitos (en especial Giardia lamblia), prueba del aliento con urea marcada con C13 y prueba del hidrógeno espirado.

La pancreatitis aguda es una enfermedad inflamatoria, causada por la liberación intersticial y, en consecuencia, la autodigestión del páncreas por sus propias 
enzimas. La causas más frecuentes son ${ }^{5}$ : enfermedades sistémicas (35\%): infecciones, enfermedades del colágeno y vasculitis; mecánico estructural (25\%): traumatismos y alteraciones u obstrucciones del árbol biliar; idiopática (25\%); otras: metabólicas, fármacos, tóxicos, hereditaria.

La clínica se caracteriza por dolor abdominal y vómitos; se asocian en el $70 \%$ de los pacientes. En casos excepcionales, el dolor abdominal puede estar ausente ${ }^{5}$. La exploración física frecuentemente muestra: dolor a la palpación del epigastrio y flancos, a menudo asociado a hipoperistaltismo, en casos graves pueden aparecer signos de abdomen agudo, distress respiratorio y los signos de Gray-Turner (oscurecimiento de la piel de los flancos) y de Cullen (oscurecimiento alrededor del ombligo).

Exploraciones complementarias: analítica sanguínea: amilasa y lipasa. El grado de amilasemia no se correlaciona con la gravedad del cuadro. La aclaración amilasa-creatinina no ofrece una mayor sensibilidad diagnóstica. Pruebas de imagen: radiografía de abdomen: tiene escasa utilidad ${ }^{5}$. Ecografía abdominal: es de utilidad en el diagnóstico y seguimiento de las complicaciones. Otras: el angio-TC y la colangiopancreatografía retrógrada son de utilidad para completar el estudio y, en algunos casos, proceder al tratamiento.

El tratamiento es hospitalario con el ingreso del paciente. Si en las primeras horas aparecen signos de gravedad, está indicado el ingreso en $\mathrm{UCl}^{5}$. El tratamiento está orientado a eliminar la causa, combatir los síntomas y evitar las complicaciones locales.

\section{Conclusión}

Para poder realizar un correcto diagnóstico de los cuadros de dolor abdominal es muy importante realizar una pormenorizada anamnesis y una cuidadosa exploración física, que nos permitan después orientar las exploraciones complementarias, si son necesarias.

En primera instancia, catalogaremos el dolor abdominal (agudo, recurrente o crónico) y descartaremos la causa orgánica del dolor mediante la exploración física y las exploraciones complementarias pertinentes, en función de los datos recogidos en la anamnesis y la exploración física. 
Bibliografía

1. Bourrillon A. Dolor abdominal. Elsevier-Masson; 2009.

2. Chitkara DK, Rawwat DJ, Talley NJ. The epidemiology of childhood recurrent abdominal pain in Western countries: a systematic review. Am J Gastroenteol. 2005;100(8):1868-75.

3. Plunkett A, Beattie RM. Recurrent abdominal pain in childhood. J R Soc Med. 2005;998(3): 101-6.
4. McOmber ME, Shulman RJ. Abdominal pain and irritable bowel syndrome in children. Curr Opin Pediatr. 2007;19(5):581-5.

5. Vilar Escrigas P, García Burriel Jl. Pancreatitis aguda. Protocolos diagnósticos y terapéuticos en pediatría. Gastroenterología [consultado el 20/10/ 2009]. Disponible en www.aeped.es/protocolos/ gastroentero/18.pdf 